\title{
Electronic Structure of $\mathrm{La}_{0.6} \mathrm{Sr}_{0.4} \mathrm{FeO}_{3}$ Thin Film by Soft-X-Ray Spectroscopy
}

\author{
$\underline{\text { T. Higuchi }}^{1)}$, M. Matsumoto ${ }^{1)}$, W. Yang ${ }^{2)}$, P. Olalde-Velasco ${ }^{2)}$, J Chen, Y. Liu ${ }^{2)}$, and J. -H Guo ${ }^{2)}$ \\ 1) Department of Applied Physics, Tokyo University of Science, 1-3 Kagurazaka, Shinjuku, Tokyo 162-8601, Japan \\ ${ }^{2)}$ Advanced Light Source, Lawrence Berkeley national Laboratory, Berkeley, CA 94720, U.S.A \\ Fax: 81-3-5228-8241, e-mail: higuchi@rs.kagu.tus.ac.jp
}

\begin{abstract}
The electronic structure of $\mathrm{La}_{0.6} \mathrm{Sr}_{0.4} \mathrm{FeO}_{3}$ thin film on $\mathrm{MgO}$ substrate has been studied using resonant soft-X-ray emission spectroscopy (SXES) and X-ray absorption spectroscopy (XAS). The thin film has the mixed valence states of $\mathrm{Fe}^{2+}$ and $\mathrm{Fe}^{3+}$ in the ground state. The valence band is mainly composed of $\mathrm{O} 2 p$ state hybridized with $t_{2 \mathrm{~g}}$ and $e_{\mathrm{g}}$ states of Fe $3 d$. The conduction band is composed of $t_{2 \mathrm{~g}}$ and $e_{\mathrm{g}}$ states of $\mathrm{Fe} 3 d$ and hole-induced state created by $\mathrm{Sr}$ doping. These findings accord with the result of electronic structure expected by tight-binding calculation that included the effect of electron-electron interaction.
\end{abstract}

Key words: $\mathrm{La}_{0.6} \mathrm{Sr}_{0.4} \mathrm{FeO}_{3}$ thin film, electronic structure, soft-X-ray emission spectroscopy (SXES)

\section{INTRODUCTION}

The perovskite-oxide $\mathrm{La}_{1-x} \mathrm{Sr}_{x} \mathrm{FeO}_{3}$ shows interesting physical properties as a function of composition [1-5]. $\mathrm{LaFeO}_{3}(x=0)$ with distorted orthorhombic structure is an antiferromagnetic insulator with a high Neel temperature $\left(T_{\mathrm{N}}=738 \mathrm{~K}\right)$. The character of the band gap is of the charge-transfer (CT) type, and optical gap is $2.1 \mathrm{eV}$ [2]. $\mathrm{SrFeO}_{3} \quad(x=1)$ with cubic structure is a helical antiferromagnetic metal with $T_{\mathrm{N}}=134 \mathrm{~K}$. The most striking behavior is observed in the intermediate composition region with rhombohedral structure. In particular, the $\mathrm{La}_{1 / 3} \mathrm{Sr}_{2 / 3} \mathrm{FeO}_{3}$ undergoes a metal-insulator (MI) transition at $\sim 200 \mathrm{~K}$, evidenced by a jump in the resistivity of more than 1 order of magnitude with decreasing temperature. ${ }^{1} \quad$ The MI transition has been classified as charge ordering, and it shows up accompanied by antiferromagnetic spin ordering below $\sim 200 \mathrm{~K}$. The charge disproportionation, which occurs from the paramagnetic average valence state $\left(\mathrm{Fe}^{3.7+}\right)$ above $\sim 200 \mathrm{~K}$ to the antiferromabnetic charge ordering state $\left(\mathrm{Fe}^{3+}: \mathrm{Fe}^{5+}=2: 1\right)$ below $\sim 200 \mathrm{~K}$, is closely related with the MI transition. This fact has been clarified by Mössbauer spectroscopy [1,6], X-ray scattering, ${ }^{7}$ and neutron diffraction. ${ }^{8}$

In recent years, the electronic structures of $\mathrm{La}_{1-x} \mathrm{Sr}_{x} \mathrm{FeO}_{3}$ and the related $\mathrm{Fe}$ compounds have refocused in research area of solid state physics [7-11]. Wadati et al. has prepared the $\mathrm{La}_{1-x} \mathrm{Sr}_{x} \mathrm{FeO}_{3}$ thin film by pulsed laser deposition (PLD) and studied its electronic structure by theoretical calculation and photoemission spectroscopy (PES) [10,11]. The valence band consists of the $t_{2 \mathrm{~g}}$ and $e_{\mathrm{g}}$ subbands of Fe $3 d$ state hybridized with O $2 p$ state. The valence band and core level shift to lower binding energy side with increasing $x$. In $x>0.4$, the $\mathrm{Fe} 3 d$ band shifts downward relative to the Fermi level $\left(E_{\mathrm{F}}\right)$ by $\sim 1.0 \mathrm{eV}$ compared with the band calculation assuming the $G$-type antiferromagnetic state and to form a gap of $\sim 1.0 \mathrm{eV}$ at $E_{\mathrm{F}}$. These findings indicate the existence of spectral weight transfer from below to above $E_{\mathrm{F}}$ across the gap. This may attribute to a strong localization effect of doped holes due to polaron formation. Therefore, the authors believe that understanding electron correlation is also one of the most important subjects for the MI transition. However, the contribution of electron correlation energy has not been directly clarified by experimental method thus far.

In this paper, we present the soft-X-ray emission spectroscopy (SXES) and X-ray absorption spectroscopy (XAS) spectra of $\mathrm{La}_{0.6} \mathrm{Sr}_{0.4} \mathrm{FeO}_{3}$ (LSFO) thin film. SXES is related directly to the occupied density-ofstate (DOS) [12-16]. SXES detects the electronic structure of the bulk state owing to the long mean free path of the soft-X-rays. The partial-DOS (PDOS) localized at an atom can be also obtained from SXES spectra, because SXES has a clear selection rule regarding the angular momentum due to dipole selection. XAS is related directly to the unoccupied DOS. This optical process is a local process because of the localized core state. It is governed by the dipole selection rules so that XAS provides spectra related to the site- and symmetry-selected DOS.

\section{EXPERIMENTAL}

LSFO thin film was deposited on an $\mathrm{MgO}$ (100) substrate by PLD using a ceramic target. The LSFO ceramics target were synthesized by a solid state reaction method and pressed into cylinders, then sintered in air at $1200^{\circ} \mathrm{C}$ for $6 \mathrm{~h}$. The target density was approximately $98 \%$.

The PLD system was arranged in a symmetric configuration with a rotating substrate holder for compositional uniformed. The base pressure was ordinary $\sim 3 \times 10^{-8}$ Torr, and the substrate was inserted from a load lock chamber to main chamber a low base pressure. A KrF excimer laser $(\lambda=248 \mathrm{~nm})$ was used as the ablation beam. The repetition frequency and laser power were $5 \mathrm{~Hz}$ and $220 \mathrm{~mJ}$, respectively [17]. The oxygen gas pressure and substrate temperature were 
fixed at $10 \mathrm{mTorr}$ and $700^{\circ} \mathrm{C}$, respectively. The film thickness was approximately $20 \mathrm{~nm}$. The prepared LSFO thin film on $\mathrm{MgO}$ (100) substrate exhibited the $c$-axis orientation.

The XAS and SXES spectra were obtained using photons from beam line BL-8.0.1 at the Advanced Light Source in Lawrence Berkeley National Laboratory [18-20]. A Rowland-circle soft-X-ray emission spectrometer with spherical gratings and a photon-counting area detector was used to obtain the SXES spectra. The resolution of the spectrometer was $\sim 0.5 \mathrm{eV}$ at $h v=700 \mathrm{eV}$. The energy axis of XAS spectrum was calibrated by measuring the XAS spectrum of Fe-metal. The energy axis of SXES spectrum was calibrated by peak position of elastic scattering, which corresponds to excitation energy.

\section{RESULTS AND DISCUSSION}

Figure 1 shows the Fe $2 p$ XAS spectrum of LSFO thin film. The spectrum consists of two parts derived from the spin-orbit split of $L_{3}\left(2 p_{3 / 2}\right)$ and $L_{2}\left(2 p_{1 / 2}\right)$ states. They are further split into the $t_{2 \mathrm{~g}}$ and $e_{\mathrm{g}}$ states due to the octahedral ligand field. The crystal-field splitting (10Dq) corresponding to the energy separation between $t_{2 \mathrm{~g}}$ and $e_{\mathrm{g}}$ states is $1.5 \mathrm{eV}$. The spectral shape, peak position, and $10 \mathrm{Dq}$ are similar to those of $\mathrm{La}_{1-x} \mathrm{Sr}_{x} \mathrm{FeO}_{3}$, which was theoretically calculated assuming a high-spin $\left[t_{2 \mathrm{~g}}\right]^{3}\left[e_{\mathrm{g}}\right]^{2}$ ground state [21].

Figure 2 shows the $\mathrm{O} 1 s$ and Fe $2 p$ SXES spectra and $\mathrm{O} 1 s$ XAS spectrum of LSFO thin film, where the abscissa is the relative energy to Fermi level $\left(E_{\mathrm{F}}\right)$. The $\mathrm{O} 1 s$ and Fe $2 p$ SXES spectra were measured at $h v=560$ $\mathrm{eV}$ and $735 \mathrm{eV}$, respectively. The $\mathrm{Fe} 2 p$ and $\mathrm{O} 1 s$ SXES spectra, which correspond to the fluorescence spectra, reflect the Fe $3 d$ and O $2 p$ PDOS, respectively, in the valence band. The PDOS cannot obtain from the PES spectra because the PES reflects the total-DOS at the surface state. Therefore, the detailed discussion of the valence band is possible in the fluorescence spectra. The energy position of $\mathrm{O} 2 p$ state overlaps with that of $\mathrm{Fe} 3 d$ state. This result indicates that the Fe $3 d$ state hybridizes with $\mathrm{O} 2 p$ state in the valence band. The valence band has three structures labeled $\mathrm{A}, \mathrm{B}$ and $\mathrm{C}$. The Fe $3 d$ contribution is more

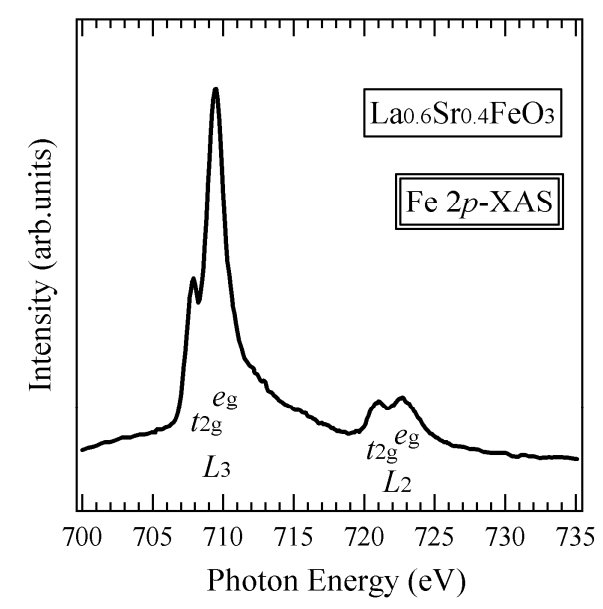

Fig. 1 Fe $2 p$ XAS spectrum of LSFO thin film.

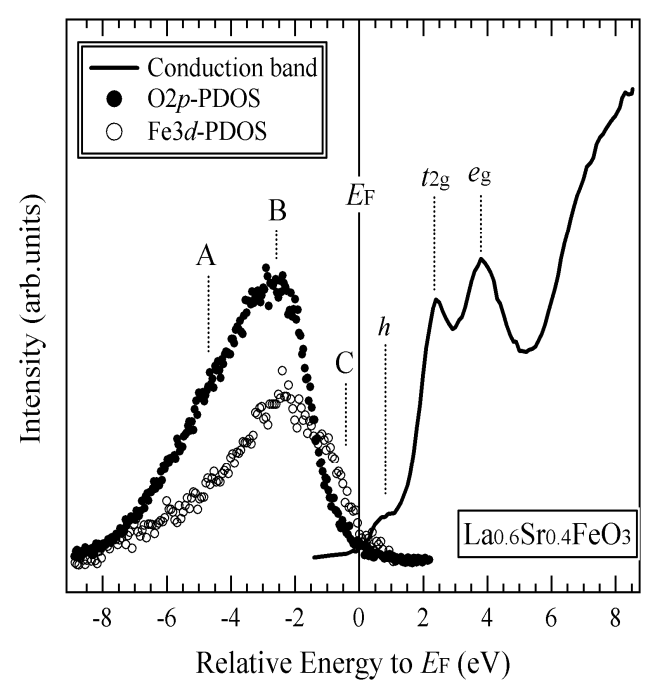

Fig. 2 Fe $2 p$, O $1 s$ fluorescence spectra and O $1 s$ XAS spectrum of LSFO thin film presented on a relative energy compared to $E_{\mathrm{F}}$.

significant in the $\mathrm{B}$ and $\mathrm{C}$ peaks. In particular, the $\mathrm{Fe}$ $3 d$ PDOS is dominantly at near $E_{\mathrm{F}}$. On the other hand, from the dipole selection rule, it is understood that the $\mathrm{O}$ $1 s$ XAS spectrum of Fe oxide corresponds to transitions from $\mathrm{O} 1 s$ to $\mathrm{O} 2 p[13,22]$. The conduction band has three structures. Two sharp peaks at $\sim 2.2$ and $\sim 3.7 \mathrm{eV}$ from $E_{\mathrm{F}}$ correspond to the $t_{2 \mathrm{~g}}$ and $e_{\mathrm{g}}$ states, respectively, of $\mathrm{Fe} 3 d$ conduction band.

In the XAS spectrum of Fig. 2, $h$ peak is observed at near $E_{\mathrm{F}}$. Similar peak has been reported in ref. 11 . This peak cannot reproduce by the tight-binding calculation. In the tight-binding calculation and spectroscopic data $[10,11]$, the value of the band gap of the parent insulator $\left(\mathrm{LaFeO}_{3}\right)$ is estimated to be $\sim 2 \mathrm{eV}$, which corresponds to the optical gap reported by Arima et al [2]. When holes $\left(\mathrm{Sr}^{2+}\right)$ are doped into $\mathrm{La}^{3+}$ site of $\mathrm{LaFeO}_{3}, E_{\mathrm{F}}$ moves downward and crosses the $e_{\mathrm{g}}$ band at $E_{\mathrm{F}}$, making the system metallic if the rigid-band model can be applied. Then, hole-induced state corresponding to peak $h$ appears above $E_{\mathrm{F}}$. The existence of the hole-induced state indicates that the rigid-band model is no more valid, and doped holes enter split-off localized states formed by hole doping.

Figure 3 shows the comparison between the Fe $2 p$ fluorescence spectrum and the theoretical Fe $3 d$ PDOS calculated by Wadati et al $[10,11]$. Wadati et al. has studied the electronic structure of LSFO thin film by PES and tight-binding calculation. His calculation is similar to local density of approximation (LDA) $+U$ in the sense that the effect of electron-electron interaction and hence the value of the band gap is adjusted via $\Delta \mathrm{E}$ in the tight-binding calculation and via $U$ in the LDA $+U$ calculation. In the PES spectra, the line shape and peak position do not accord with the tight-binding calculation. This may originate other interaction for photoemission process. However, the spectral shape of Fe $3 d$-PDOS in the valence band obtained by the tight-binding calculation accords with the Fe $2 p$ fluorescence spectrum. The magnitude of electron correlation energy is approximately $3.0 \mathrm{eV}$, which has estimated 


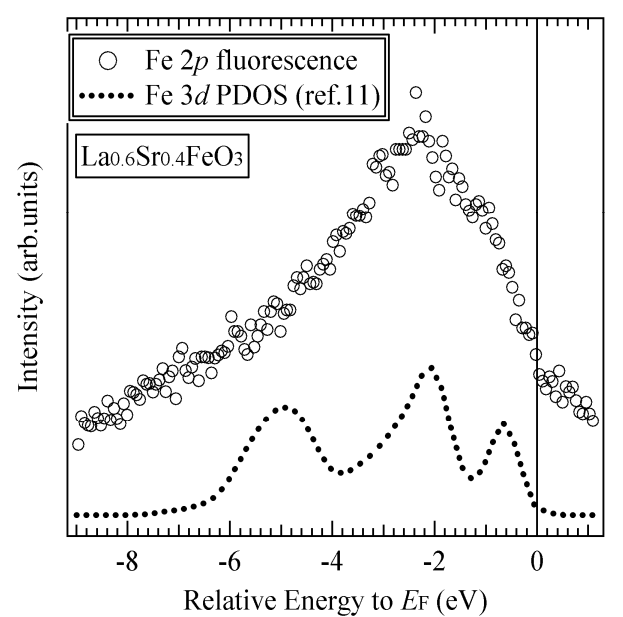

Fig. 3 Comparison of Fe $2 p$ fluorescence spectrum measured at $h v=735 \mathrm{eV}$ and Fe $3 d$ PDOS curves calculated by Wadati et al $[10]$.

from tight-binding calculation. This value accords with the energy separation between $\mathrm{C}$ peak and $t_{2 \mathrm{~g}}$-subband in Fig. 2. The existence of $\mathrm{Fe} 3 d$ PDOS at $E_{\mathrm{F}}$ has an important role for the MI transition of $\mathrm{La}_{1-x} \mathrm{Sr}_{x} \mathrm{FeO}_{3}$. Because the bottom of the conduction band is also $\mathrm{Fe} 3 d$ state, the electrical properties contribute to the magnitude of electron correlation energy. Thus, the SXES spectra in this study give an useful information for electron correlation.

\section{CONCLUSION}

The authors have studied the electronic structure of LSFO thin film using SXES and XAS. The conduction band consists of the $t_{2 \mathrm{~g}}$ and $e_{\mathrm{g}}$-subband of Fe $3 d$ state and the hole-induced state near $E_{\mathrm{F}}$ created by $\mathrm{Sr}$ doping. The valence band is mainly composed of the $t_{2 \mathrm{~g}}$ - and $e_{\mathrm{g}}$-subband of $\mathrm{Fe} 3 d$ state hybridized with $\mathrm{O} 2 p$ state. The spectral shape and peak position of valence band are in good agreement with the result of electronic structure expected by tight-binding calculation that included the effect of electron-electron interaction.

\section{ACKNOWLEDGEMENTS}

This work was partly supported by the Grant-in-Aid for Science Research C (No. 22560670) from the Ministry of Education, Culture, Sports, Science and Technology in Japan.

\section{REFERENCES}

[1] M. Takano, J. Kawachi, N. Nakanishi, and Y. Takeda, J. Solid State Chem. 39 (1981) 75.

[2] T. Arima, Y. Tokura and J. B. Torrance, Phys. Rev. B 48 (1993) 17006.

[3] M. Imada, A. Fujimori, and Y. Tokura, Rev. Mod. Phys. 70 (1998) 1039.

[4] J. Matsuno, T. Mizokawa, A. Fujimori, K. Mamiya, Y. Takeda, S. Kawasaki, and M. Takano, Phys. Rev. B 60 (1999) 4605.

[5] S. K. Park, T. Ishikawa, Y. Tokura, J. Q. Li, and Y. Matsui, Phys. Rev. B 60 (1999) 10788.

[6] P. D. Battle, T. C. Gibb, and P. Lightfoot, J. Solid State Chem. 84 (1990) 237.

[7] J. Herrero-Martin1, G. Subias, J. Garcia, J. Blasco, and M. C. Sánchez, Phys. Rev. B 79 (2009) 045121.

[8] J. B. Yang, X. D. Zhou, A. Chu, W. M. Hikal, Q. Cai, J. C. Ho, D. C. Kundaliya, W. B. Yelon, W. J. James, H. U. Anderson, H. H. Hamdeh, and S. K. Malik, J. Phys.: Condens. Matter 15 (2003) 5093.

[9] A. Chainani, M. Mathew, and D. D. Sarma, Phys. Rev. B 48 (1993) 14818.

[10] H. Wadati, D. Kobayashi, H. Kumigashira, K. Okazaki, T. Mizokawa, A. Fujimori, K. Horiba, M. Oshima, N. Hamada, M. Lippmaa, M. Kawasaki, and H. Koinuma, Phys. Rev. B 71 (2005) 035108.

[11] H. Wadati, A. Chikamatsu, M. Takizawa, R. Hashimoto, H. Kumigashira, T. Yoshida, T. Mizokawa, A. Fujimori, M. Oshima, M. Lippmaa, M. Kawasaki, and H. Koinuma, Phys. Rev. B 74 (2006) 115114.

[12] A. Kotani and S. Shin, Rev. Mod. Phys. 73 (2001) 203.

[13] J. C. Fuggle and J. E. Inglesfield, "Unoccupied Electronic States" Springer-Verlag (1991).

[14] J. Nordgren and R. Nyholm, Nucl. Instrum. Methods Phys. Res. A 246 (1986) 242.

[15] J. Nordgren, G. Bray, S. Cramm, R. Nyholm, J. E. Rubensson, and N. Wassdahl, Rev. Sci. Instrum. 60 (1989) 1690.

[16] J.-H. Guo, Y. Luo, A. Augustsson, J.-E. Rubensson, C. Såthe, H. Ågren, H. Siegbahn, and J. Nordgren, Phys. Rev. Lett. 89 (2002) 137402.

[17] N. Sata, K. Ikeda, F. Iguchi, and H. Yugami, Solid State Ionics 178, 1563 (2007).

[18] J. J. Jia, T. A. Callcott, J. Yurkas, A. W. Ellis, F. J. Himpsel, M. G. Samant, J. Stöhr, D. L. Ederer, J. A. Carlisle, E. A. Hudson, L. J. Terminello, D. K. Shuh, and R. C. C. Perera, Rev. Sci. Instrum. 66 (1996) 1394.

[19] J. -H. Guo, S. M. Butorin, N. Wassdahl, J. Nordgren, P. Berastegut, and L.-G. Johansson, Phys. Rev. B 61 (2000) 9140.

[20] M. Magnuson, S. M. Butorin, J. -H. Guo, and J. Nordgren, Phys. Rev. B 65 (2002) 205106.

[21] M. Abbate, F. M. F. de Groot, J. C. Fuggle, A. Fujimori, O. Strebel, F. Lopez, M. Domke, G. Kaindl, G. A. Sawatzky, M. Takano, Y. Takeda, H. Eisaki, and S. Uchida, Phys. Rev. B 46 (1992) 4511.

[22] T. Higuchi, Y. Liu, P. Yao, P. Glans, J.- H. Guo, C. Chang, Z. Wu, W. Sakamoto, N. Itoh, T. Shimura, T. Yogo, and T. Hattori, Phys. Rev. B 78 (2008) 085106. 